\title{
THE DEVELOPMENT OF EARLY \\ SYMBOLIZATION: EDUCATIONAL IMPLICATIONS
}

\author{
JUDY S. DELOACHE,* DAVID H. UTTAL and SOPHIA L. \\ PIERROUTSAKOS
}

University of Illinois, Urbana-Champaign, USA

\begin{abstract}
Research on the symbolic functioning of very young children has important implications for educational materials. We argue that there are no transparent symbols; one can never assume that what seems to be an obvious symbolic relation is obvious to young children. We have discovered that young children have particular difficulty understanding and using symbols that are themselves interesting objects. A symbol, such as a scale model of a room, that is salient and appealing as an object, requires a dual representation: To use a model, one must simultaneously represent both the model itself and its referent. Research on young children's understanding and use of models indicates that they have particular difficulty achieving dual representation. This work has clear implications for the use of symbolic objects for educational purposes. We discuss several examples of commonly used symbolic objects, suggesting that they may be less helpful to young learners than is generally assumed. () 1998 Elsevier Science Ltd. All rights reserved.
\end{abstract}

\section{Introduction}

As members of human societies, children's initial exposure to symbols comes before birth with the words and music they hear in the womb. From birth on, they are immersed in an increasingly complex and extensive web of cultural symbols. During the first few years of life, the capacity for symbolic production and comprehension expands prodigiously.

There are numerous different types of symbols. Some, such as some religious icons, are primarily intended to be emotionally evocative, and they have no specific content. Others, such as alphabet letters and numerals, have no inherent content or meaning, but convey information when combined in systematic ways. Yet other symbols, such as maps

*Address for correspondence: Department of Psychology, University of Illinois, 603 E. Daniel St., Champaign, IL 61820 USA. 
and photographs, are highly specific representations of real entities; they themselves contain and can convey specific information.

Informational symbols are routinely employed by teachers and other speakers and writers in an effort to maximize communication. Pictures, maps, models, diagrams, and graphs are used to present information as clearly as possible. Are such symbolic aids of educational benefit? The position we advance in this paper is that it depends both on the nature of the educational aid and on the characteristics of the audience, and, most crucially, on the interaction between the two.

We will summarize research with infants and very young children showing that understanding and using symbols is a surprisingly difficult task. It is generally recognized that abstract, arbitrary symbol systems, such as numbers and letters, are difficult for children to master, and that the first step in achieving mastery is the insight that these squiggles on a page carry meaning, that they stand for something. It is less generally accepted, however, that the same is true of all symbol systems. The main point of this article is that no symbol system is fully transparent: It cannot be assumed that even the most iconic symbol will automatically be interpreted as a representation of something other than itself.

We will focus especially on one hypothesis about what makes early symbol use so problematic, and we will argue that the same difficulties that beset very young children's symbol use continue to undermine school age children's ability to profit from a variety of communicative symbols. This position has clear implications for the design and use of educational materials, and they will be discussed in the final section of the paper.

\section{Research on Symbolic Functioning}

In all our research, we focus on symbols that convey information, that is, symbols from which one can learn something new. By examining a map of Greece, for example, one can learn the positions of Thessaloniki and Athens, the distance between them, how far they are from the sea, and so forth. Virtually all graphs, diagrams, and maps are informational symbols, but pictures and models can also serve an informative function.

To use an informational symbol successfully, one must appreciate the relation between it and its referent in at least three ways. First, one must realize that a symbol-referent relation exists. The map of Greece, for example, is useful only if one realizes what a map is, that it represents a particular part of the world. Second, one must understand something about how the symbol is related to its referent. With respect to the map of Greece, one must know that cities are represented by circles, that the blue areas stand for seas, that the relative spatial relations among the real entities are preserved on the map, that it is drawn to scale, and so forth. Finally, using an informational symbol requires computing specific relations between the symbol and its referent. The erstwhile traveler in Greece must associate the upper and lower circles and the right and left blue areas on the map with the corresponding real cities and with the Aegean and Ionian Seas.

Do children have to learn to interpret and use informational symbols? It is often assumed that learning is not required for highly iconic symbols such as photographs or realistic models, that is, symbols that resemble their referents. One reason for this assumption is the fact that even infants can recognize pictured information. For example, several studies have established that if young infants are familiarized with a three-dimensional object or 
a real person, they can subsequently recognize a two-dimensional depiction of that thing or person (e.g., DeLoache, Strauss, \& Maynard, 1979; Dirks \& Gibson, 1977).

Perceiving similarity between a picture and its referent is not, however, the same thing as understanding the nature of the picture. In the following section, we summarize some recent and ongoing research with infants in our lab that supports this assertion. We include these infant studies in this paper on educational issues to emphasize how knowledge about the nature and meaning of symbols — even the most iconic and realistic ones — is never automatic.

\section{Pictures}

We have recently completed two studies of a phenomenon that has often been mentioned in anecdotes, but that has never before been systematically investigated. The phenomenon of interest is behavior by infants and very young children that appears to involve treating a pictured object as though it were a real object (for example, attempted grasping and other manual behaviors directed at depicted objects) (Murphy, 1978; Ninio \& Bruner, 1978).

In our first study (DeLoache, Pierroutsakos, Uttal, Rosengren \& Gottlieb, 1998), we examined 9-month-old infants' manual responses to pictures, focusing particularly on behaviors that appeared to be attempts to grasp or pick up the depicted objects. We presented the infants with specially constructed picture books with a highly realistic color photograph of a single object on each page. The pictured objects were small enough (ca. $3.8 \times 2.5 \mathrm{~cm}$ ) that a 9-month-old could have picked them up, had they been real objects.

The results were surprisingly clear: Every infant attempted, at least once or twice, to grasp or pick up a pictured object. Some babies were remarkably persistent, making repeated and concerted efforts to pluck the object from the page.

In a second study, we again tested 9-month-olds and also a group of 15-month-olds. Some infants received the same "baby-sized" pictures as were used before; others received pictures in which the depicted object was larger $(\mathrm{ca} .8 .9 \times 5.1 \mathrm{~cm}$, a size of real object that would be more difficult for young infants to grasp). Preliminary results indicate that the frequency of grasping attempts declines with age and that more efforts are directed to the smaller, "graspable," objects than to the larger ones.

We conclude that the infants in our studies do not fully understand the nature of pictures. This is in spite of the fact that even younger infants can recognize a picture of a familiar object and discriminate it from a novel one, and they can also discriminate between a real object and a picture of it (DeLoache, et al., 1979). Nevertheless, our subjects still lack an understanding of an important feature of pictures - the fact that pictured objects are not tangible.

This research highlights the fact that understanding the nature of a symbol - even one so simple and iconic as a highly realistic color photograph — is not automatic. In the research review that follows, we document that problems with understanding symbols continue well beyond infancy. Like infants, young children often respond to a symbol more in terms of the object itself than in terms of what it represents. 


\section{Scale Models}

Much of our research on early symbolic functioning has focused on very young children's understanding and use of scale models. In this research, a model gives the child information about a full-sized room that the model represents. If children recognize the model-room relation, they should be able to apply the information they gain from the model to the room itself.

In this research, young children (2.5- and 3.0-year-olds) participate in a simple objectretrieval game in which they must find an attractive toy that is hidden in a natural location in a room. At the beginning of the session, the children are given an orientation in which they are explicitly told about the correspondence between a scale model of the room and the room itself. Both the model and the room contain several items of furniture (couch, chairs, table, etc.) with a high degree of surface similarity (e.g., same color, fabric). The child watches as the experimenter hides a small toy somewhere in the scale model. The child is instructed that a larger toy is hidden in the same place in the room.

Each trial consists of three parts: the hiding event in the model, the retrieval of the large toy in the room (retrieval 1), and the retrieval of the miniature toy in the scale model (retrieval 2). Success on retrieval 1 is taken as evidence that the children recognize the model-room relation. The purpose of the second retrieval is to make sure that they remember the location of the small toy they saw hidden in the model; if they do, their failure to find the larger toy in the room could not be due to forgetting the information learned from the model.

The performance of 2.5- and 3.0-year-old children is dramatically different in this task. Figure 1 shows the results from the original study (DeLoache, 1987). The errorless retrieval rate for retrieval 1 was $77 \%$ for the 3.0 -year-old children, but only $15 \%$ for the 2.5-year-olds. The 3.0-year-olds used the information from the model to locate the larger toy in the room. The 2.5-year-olds gave no evidence of realizing that the model and room were related. This difference cannot be attributed to memory or motivational differences, because retrieval 2 performance was the same for the two age groups.

It should be noted that the 2.5-year-olds understood everything about this task except the critical fact that the room and the model were related. They realized they were to search for the toy hidden in the room, but they apparently did not realize they had information to lead them to the correct location.

Why is it so difficult for these young children to understand the model-room relation? The dual representation hypothesis has been proposed to account for these results (DeLoache, 1987, 1991, 1995a, b). An important factor in these tasks is the dual nature of models: While they serve as representations of a larger space, they are also objects themselves. A child, in performing the model task, must deal with this dual nature and respond to the model both as an object (a miniature room in which things can be hidden) and at the same time as a symbol that represents something other than itself (the larger room and its hiding places).

The results indicate that the 2.5-year-olds had no difficulty responding to the model as an object. After seeing a small toy hidden in it, they were successful in subsequently retrieving that object. However, they apparently failed to comprehend the representational nature of the scale model.

According to the dual representation hypothesis, the fact that the model is a highly 


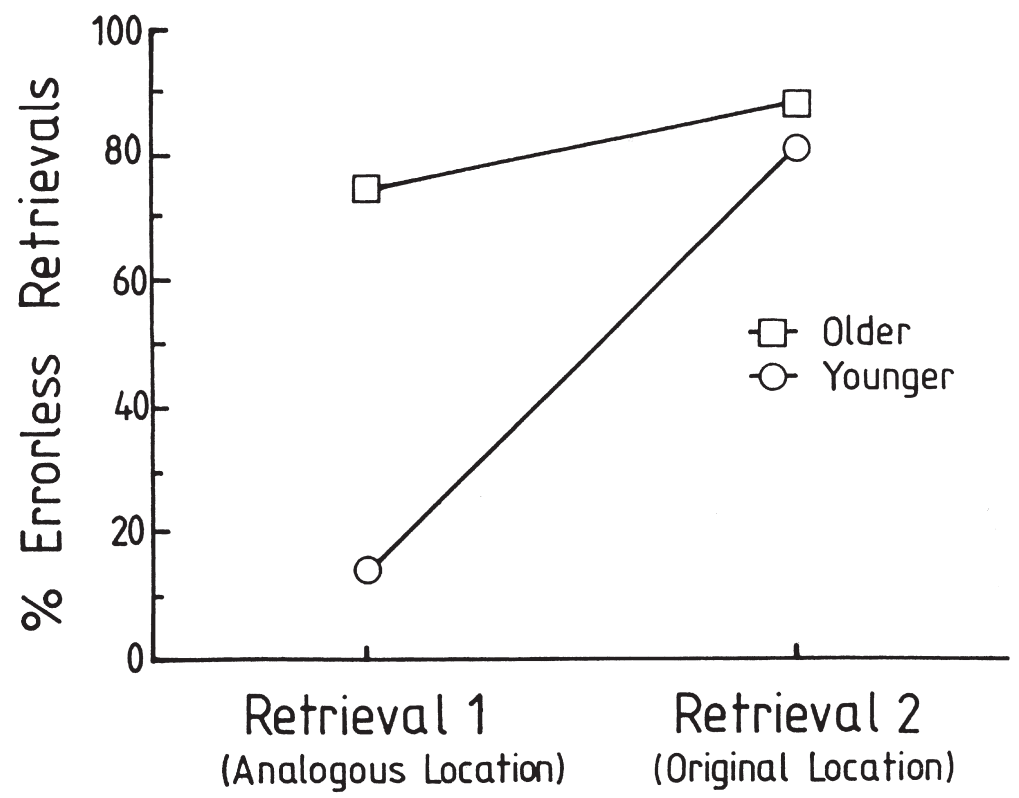

Figure 1. Children's performance in the original model study. From "Rapid change in the symbolic functioning of very young children" by Judy S. DeLoache, 1987, Science, 238, p. 1556. Copyright 1987 by the American Association for the Advancement of Science. Reprinted by permission.

attractive and salient object prevents the 2.5-year-old children from simultaneously appreciating it as a representation of the room. Their attention is directed to the model as object, and they are typically very interested in it. That attention and interest makes it very difficult for them to see it in relation to something else. Hence, they perform well with the model itself (retrieval 2), but not with the model as a source of information about the room (retrieval 1).

According to this hypothesis, 2.5-year-old children might be more successful if one could provide the same information about the location of the toy in the room in some way that did not necessitate achieving a dual representation. In other words, they should benefit from the same information provided by a medium that is not itself attractive and interesting.

Pictures are such a medium. Even though they are real objects, they are very simple and boring as objects. Furthermore, even 2.5-year-old children know that pictures primarily serve to represent something else (DeLoache \& Burns, 1993, 1994). Since children are used to thinking only of what a picture depicts, they do not have to suppress a strong response to it as an object. This should leave them free to focus on its symbolic nature.

The following prediction was made: 2.5 -year-olds should more successfully retrieve objects hidden in the room when given the location information via pictures of the room than via a scale model of the room. This prediction is counterintuitive based on a large amount of research showing that better cognitive performance is achieved with real objects than with pictures (e.g. Daehler, Lonardo, \& Bukatko, 1979; DeLoache, 1986; Sigel, 1953; Sigel, Anderson, \& Shapiro, 1966). However, the prediction does follow from the dual representation hypothesis. 
To test it, three studies compared performance in the model task versus a similar task with pictures. 2.5-year-old children participated in two-tasks, with the order counterbalanced across subjects. On one day, they participated in the standard model task in which the experimenter hid the miniature toy in the model and then asked the child to search for the larger toy in the room. On the other day, they participated in a picture task in which the experimenter pointed to a picture to indicate the location of the toy in the room. In one study, four color photographs were used, each depicting a particular hiding place in the room. In another study, a wide-angle photograph and a line drawing depicting the room were used. In both studies, 2.5-year-olds' performance was significantly better when the information was given via the picture. These young children were able to use the information given to them by the pictures to retrieve the toy, even though they failed to use the same information given via the scale model. These results support the dual representation hypothesis: Pictures alleviate the difficulty young children have in simultaneously representing a concrete object (the model) and what it represents (the room).

However, the picture task does differ from the model task in that the information is conveyed by pointing to the picture in the former, whereas the miniature toy is actually hidden in the latter. Consequently, the standard model task may make spatial relational demands that the picture task does not. To assure that the difference in results was due to the difference in medium (picture vs. model) rather than method (hiding vs. pointing), an experiment with four conditions was conducted: (1) Hide-Model, a standard model task in which the miniature object was hidden, (2) Point-Picture, a picture task replication in which the experimenter pointed to the correct one of four pictures, (3) Point-Model, in which the experimenter simply pointed to the correct hiding place in the model, and (4) Hide-Picture, in which the miniature toy was hidden behind one of the four pictures. If the children were originally hindered by their inability to represent the hiding event, rather than by the model itself, their performance should be better in the Point-Model condition when pointing is used than in the standard model task (Hide-Model).

The results of this study replicated earlier studies in that performance was good in the Point-Picture and poor in the Hide-Model condition. In the crucial condition, Point-Model, performance was low, in fact identical to that in the standard model task (Hide-Model). The young children did not know where to look for the toy when using the scale model, regardless of whether the information was given by hiding or pointing. We can thus conclude that the method of designating the correct location - hiding vs. pointing — is not responsible for the superiority of pictures over model as a source of information.

The results of this study supported the dual representation hypothesis, in that the picture superiority effect was replicated and clarified. Another result from this study provided especially strong and interesting support for the hypothesis. The fourth condition, HidePicture, proved to be incomprehensible to the 2.5-year-old subjects. When children saw the miniature toy hidden behind the appropriate picture, they apparently had no idea of its significance. There were no errorless retrievals of the toy in the room. Why was this condition so mystifying?

We believe that it had two highly problematic features. First, it violated what these young children had learned about pictures, that is, that pictures are not real objects and are not to be treated like real objects. Second, it required a dual representation, in that the children had to respond both to information contained in the pictures and at the same time to the pictures themselves as physical hiding places for the miniature toy. The children 
managed to treat the pictures as objects; they were very successful at retrieving the miniature toy from behind them. They failed, however, to treat the pictures as symbols at the same time. Responding to the pictures as hiding locations, they did not use the depicted information about the relevant location in the room. Thus, we again see young children responding to an object that has symbolic content only in terms of the object itself and not, as called for, also in terms of what it represents.

It follows from the dual representation hypothesis that decreasing the salience of a symbol as an object should make it easier for young children to detect its relation to its referent. Accordingly, in a direct test of the hypothesis, the salience of the model as an object was diminished by placing it behind a window. Neither the child nor the experimenter touched the model or its contents. The experimenter indicated the hiding location by pointing to the appropriate location in the model. The counterintuitive prediction was made that 2.5-year-old children would be more successful at retrieving the toy hidden in the room in this no-access, window condition than in the standard model task. Our reasoning was that if the children never touched the model or retrieved the miniature toy from it, the model would be less salient as an object. Hence, it would be easier for it to serve as a symbol.

The results confirmed the prediction. Our subjects were significantly more successful when they had no access to the model as an object (window condition) than when they did have access to it (standard model task).

In a second direct test of the dual representation hypothesis, we did the opposite; we attempted to increase the salience of the model as an object, predicting that performance would be poorer as a consequence. The subjects were 3-year-olds, the age group that typically succeeds in the standard model task. To increase salience, we simply gave the children extra experience with the model. They were encouraged to play with the model and its contents for five to ten minutes before we began the standard model task.

The predicted results were again obtained. The 3-year-olds who had extra time physically manipulating the model were less successful at using it as a source of information for the room than were children who did not have this extra experience. The errorless retrieval rate was significantly lower in the experience condition than in the standard task. The results, like those for pictures and the model behind the window, provided strong support for the dual representation hypothesis.

We now describe the most stringent test of this hypothesis to date. This study (DeLoache, Miller, \& Rosengren, 1997) clearly demonstrates that it is specifically the representational feature of a scale model that is problematic for very young children.

We convinced 2.5-year-old children that we were shrinking a room. This rather peculiar experimental manipulation was done with the goal of removing the representational nature of the task. Our reasoning was that if the children believed that the model actually was the room, then no dual representation would be required.

We used an artificial room $($ a $1.85 \times 2.57 \times 1.88 \mathrm{~m}$ room constructed of white fabric walls) and a scale model of that room, both of which have been used in several previous model studies. In the orientation phase of the study, 2.5-year-old children were introduced to a troll doll, to the troll's room (the portable room), and to a "shrinking machine." The child was told that the machine could "shrink toys." To demonstrate, the experimenter pointed the machine at the doll and "turned it on." The child and experimenter then waited in an adjoining room, listening to the sounds of the shrinking machine (actually a tape 
recording of computer-generated tones). When they re-entered the lab room, the child discovered a miniature troll in place of the larger toy that had been there before. Next, the doll was "enlarged" by the machine, and the artificial room was shrunk and then enlarged again.

On the first experimental trial, the troll was hidden in the artificial room, the shrinking machine was aimed at the room, and the child and experimenter again waited in the adjoining room. Upon entering the lab, the child found the scale model situated in the middle of the space that had been occupied by the artificial room. The child was encouraged to find the troll, which was, of course, hidden in the model in the place that corresponded to where the larger doll had been hidden in the room. On the next trial, the child watched as the miniature troll was hidden in the model, waited while the room was "blown up" by the machine, and then searched in the room. Subsequent trials alternated between shrinking and enlarging events.

Before describing the results of the study, we should emphasize that the children did appear to believe that we really were shrinking and blowing up the troll and the room. Both the two experimenters and the parents rated the children as being convinced that this was the case.

The results were as predicted. The 2.5 -year-olds in the shrinking room condition performed $76 \%$ errorless retrievals, a rate significantly better than that of two different control groups using the same artificial room and model.

We conclude that the typical failure of 2.5-year-old children in the standard model task is due to its representational nature and specifically to the need for dual representation. When there is an identity relation between the room and model, these children are successful at applying what they know about one space to the other. When there is a symbolic relation between the two so that dual representation is required, they fail.

The dual representation hypothesis has thus received strong empirical support. Four highly counterintuitive predictions were made and confirmed: (1) two-dimensional pictures are a more useful source of information for 2.5-year-old children than three-dimensional models; (2) restricting young children's access to a model makes them better able to exploit its symbolic content; (3) providing extra access to a model renders its symbolic content less obvious to 3-year-olds; and (4) removing the symbolic nature of the model task (via the bizarre "incredible shrinking room" manipulation) makes it easier for 2.5year-olds to apply information gained from the model to the room.

We emphasize the counterintuitive nature of these results in part to highlight the strength of the support for the dual representation hypothesis. We also emphasize them to highlight the fact that adult intuitions about the symbolic functioning of young children are very poor. Adults are so steeped in symbols that they frequently fail to realize that the symbolreferent relations that are so obvious to them are not at all obvious to someone with less experience using symbols. In the following section, we consider the implications of our research on early symbolization for the use of informational symbols in education, stressing the potential fallibility of adult intuitions about what symbolic aids would be useful teaching tools. 


\section{Educational Implications}

Our research on the development of symbolization and particularly the dual representation hypothesis raises some provocative ideas about the use of symbolic aids in teaching children facts and concepts. Informational symbols in education can be very useful, but they can also engender unexpected problems parallel to those that younger children face in the model task.

An example of some of the counterintuitive problems that may arise when symbols are used in education comes from the television show Sesame Street. Many parents are happy or even proud that their children watch the show because it attempts to convey valuable information while simultaneously entertaining children. An enduring (and endearing) characteristic of the show is that normally abstract or even dry concepts are brought to life letters dance, numbers jump, and words take on the shape of the objects they represent. For example, one segment of a recent show was a beauty pageant for letters. The contestants appeared garbed in ball gowns, jewelry, and flowing tresses. Although the letter S had prevailed in the swimsuit competition, E was the overall winner. After E was crowned, the master of ceremonies sang a celebratory song, praising $\mathrm{E}$ for being used in so many words.

What might a toddler or preschooler take away from this show? Parents and educators would hope that the child learned something about individual letters and about the relations between letters and words. Our research on children's understanding of models, however, leads us to think twice about the utility of making letters come alive, sing, and participate in beauty pageants.

Specifically, our findings regarding the problem of dual representation suggest that children may have more difficulty using symbols that are highly attractive or salient as objects in themselves. To become literate, children must treat letters primarily as symbols of written and spoken language. They must realize, just like children in our model task, that the properties of the symbols are less important than what they represent. Focusing on the properties of the letters themselves (such as the curvaceous shape of the letter S) may make it harder for young children to interpret them as symbols. Such an effect would be analogous to what happened when we allowed children to play with the model before we asked them to use it as a symbol of the room: Playing with the model increased its salience as a thing in itself, and the children therefore had more difficulty using it as a symbol. Treating letters as toys or contestants in a beauty pageant converts highly abstract symbols into concrete objects, probably making their meaning less, rather than more, clear to very young children.

This comment on Sesame Street is of course speculative, and we do believe that children can learn from watching it. There is, however, evidence that children may have difficulty separating the characteristics of letters and words from the things in the world that they represent. Bialystok $(1991,1992)$ has shown that many children assume that there should be correspondences between characteristics of letters or words and the objects or concepts that they represent. They may think that there is a relation between the size of a word (i.e., the number of letters) and the size of the object that the word represents. For example, when asked to place cards containing either the printed words BANANA or CAR beside pictures of the appropriate referents, many children incorrectly based their judgments on the relative sizes of the objects. They assumed that BANANA should be placed beside the picture of a car, because BANANA is a long word and cars are large objects. These 
kinds of errors demonstrate that young children can easily confuse properties of a symbol and its referent when they are first learning to use a symbol system.

The difficulties that children have using symbols are not limited to letters. Examples from several domains illustrate that the use of symbols in education may not always have the intended result and that teachers should be alert to the possible difficulties that children may encounter when using symbols.

\section{Manipulatives and Mathematics Education}

Learning mathematics is a challenging task for most elementary school children. To succeed, children must grasp abstract concepts and logic. To help them, educators often use manipulatives — hand held objects that are designed to make abstract ideas more tractable. Some kinds of manipulatives, such as Dienes blocks or Cuisinaire Rods, are standardized and designed specifically to be used in mathematics instruction. In addition, teachers often use common classroom or household objects to serve the same purpose.

Our symbolization research leads us to expect that manipulatives might not always have the intended consequences, and that children might have difficulty understanding and using them. The potential problem is again dual representation: Children must simultaneously treat the manipulative as a thing in itself and a representation of something else - in this case, a mathematics fact or concept. There is no guarantee that they will readily see the symbolic relation between the manipulative and what it is intended to represent. Moreover, they may experience particular difficulty using manipulatives that are attractive, interesting or otherwise salient.

Despite the widespread use of manipulatives, there has been relatively little systematic research on their effectiveness. However, there is some evidence to support our suggestion that the link between manipulatives and what they are intended to represent is not always transparent or even easy to grasp.

Several studies have demonstrated that simple manipulatives do not necessarily help children acquire a particular concept (Goswami, 1992). For example, Hughes (1986) found that 5- to 7-year-olds had difficulty using small blocks to represent a single number (5) or simple addition problems $(4+3=7 ; 6-2=4)$. The children were told to use the blocks to show the experimenter what was printed on a card - to show what the problem meant. Their performance was quite bad - less than one-third consistently used the blocks to represent the problems. The types of errors that the children made suggested that they did not understand how the blocks were supposed to relate to the numbers and problems. For example, some children used the blocks to make a copy of the plus or minus sign, rather than to represent the answers to the problems.

These results demonstrate that being able to count blocks or other manipulatives does not mean that children can use them symbolically to solve problems. The children in this research were not helped to solve the addition and subtraction problems, because they did not understand how to use the blocks as representations of those problems.

Our perspective on the development of symbolization and the problem of dual representation leads to another counterintuitive suggestion regarding mathematics manipulatives: The best manipulatives may be those that are least interesting as objects in themselves. 
To use something as a symbol, children must focus on what the symbol represents, not on the symbol itself. Consequently, objects that are particularly interesting or attractive may be the most difficult for young children to use as symbols.

There is some suggestion that this is indeed the case. In Japan, where students excel in mathematics, a single, small set of manipulatives is used throughout the elementary school years. Because the objects are used repeatedly in various contexts, they presumably become less interesting as things in themselves. Moreover, children become accustomed to using the same manipulatives to represent different kinds of mathematics problems. For these reasons, they are not faced with the necessity of treating an object simultaneously as something interesting in its own right and a representation of something else.

In contrast, American teachers use a variety of objects in a variety of contexts. This practice may have the unexpected consequence of focusing children's attention on the objects rather than on what the objects represent. Stevenson \& Stigler (1992), who have conducted extensive research on cultural differences in mathematics achievement and instruction, have observed the following:

Japanese teachers...use the items in the math set repeatedly throughout the elementary school years...American teachers seek variety. They may use Popsicle sticks in one lesson, and marbles, Cheerios, $\mathrm{M}$ and M's, checkers, poker chips, or plastic animals in another. The American view is that objects should be varied in order to maintain children's interest. The Asian view is that using a variety of representational materials may confuse children, and thereby make it more difficult for them to use the objects for the representation and solution of mathematics problems. Multiplication is easier to understand when the same tiles are used as were used when the children learned to add. (pp. 186-187)

\section{Learning Geography: Maps Are Not Transparent Symbols of the World}

Problems using symbols in educational contexts are not limited to mathematics. Similar difficulties arise in geography instruction. Maps are the primary tool for teaching geography, and children therefore must understand that and how maps represent geographic areas. Nevertheless, there has been relatively little research on young children's understanding of maps as symbols. The research that has been conducted reveals that the relation between maps and their referents is not always transparent or even easy for children to grasp.

For example, Liben \& Downs $(1989,1992)$ asked preschoolers and young elementary school children to describe and explain various features on geographic maps, such as Rand McNalley road maps. In one task, children were asked to identify and explain the meaning of some of the symbols that appeared on the maps, including the colored lines used to represent roads of different size and quality and the compass that represents directions.

Children's responses revealed that they did understand the basic purpose of maps. For example, most children were able to identify large, blue areas on the maps as bodies of water. However, very interesting errors emerged when they were asked to identify more complex items, such as roads. One child, for example, said that a red line on the map could not be a road because there are no red roads in the world. Another child said that the red line could not be a road it was too narrow to accommodate a car. Similarly, a child who had correctly identified a blue area as water said that the compass on the map was the "lifeguard's chair" at the beach his family often visited.

Children's errors in interpreting maps therefore provide another example of the tendency to reify symbols - to assume that symbols must share properties of the things they rep- 
resent. Just as young children think a big word should represent a big object, they also believe that all symbols on maps should share the physical properties of the things they represent. Children's strong tendency to reify symbols suggests that the arbitrary nature of many symbol systems may be particularly difficult for them to grasp. Our model research has revealed that physical similarity can help young children understand the basic, representational relation between a model and its referent (DeLoache, Kolstad, \& Anderson, 1991). However, successful, flexible use of symbols requires that children eventually lose this dependence on physical similarity. Neither letters nor mathematical symbols, for example, look at all like their referents.

Maps present a particularly difficult challenge in this regard. The cartographer's choice of how to represent characteristics of the world is sometimes influenced by a desire to achieve some degree of physical similarity. Water, for example, is usually blue, both on maps and in the world. In other cases, however, there is no physical similarity between the map symbol and what it represents in the world. The red road on the map proved so challenging to children because its relation to its referent, a superhighway, is arbitrary. Children apparently have difficulty understanding that most map symbols do not look like the things they represent (Liben \& Downs, 1989).

Children's difficulties with maps also point out another important consideration regarding symbols in education: Children's symbol use may be affected or constrained by their knowledge and understanding of what the symbol is intended to represent. Consider the child who, after correctly identifying a blue area as water, said that the compass on the map was the lifeguard stand. This child clearly had some idea of the basic purpose of maps - to communicate information about places. However, his understanding of exactly what the map was intended to communicate was influenced greatly by his own experiences in the world. For him, the most salient body of water was a local beach, and the compass on the map reminded him of the lifeguard stand at the beach.

Adults know that we need maps to communicate information about the geographic world - which cities are located in which states, for example, or how to travel between cities. Children may have little understanding of the man-made, geographical organization of the world. They may not know, for example, that cities are contained within countries, and that countries are contained within continents. Lacking a full understanding of what the map was intended to communicate, the child may assume that it communicates what he knows best - a local, highly familiar area. This example illustrates that to use symbols effectively in education, we must consider what children know about the information that a given symbol is intended to communicate. Children probably know little about the Interstate Highway System, for example. We consequently should not be surprised if they fail to understand a symbol that is intended to represent an interstate.

A final example illustrates a similar point. Vosniadou \& Brewer (1992) have shown that children's conceptual models of the earth are highly influenced by the symbol, the globe, that is used to represent it. However, the information that children gain from studying a globe must be integrated with what they gain from their everyday, perceptual experience. They must reconcile two facts: The earth looks flat (e.g., ships seem to fall off the horizon), but the symbol that is used to represent the earth is a sphere. These seemingly incongruous observations get resolved in some very interesting ways. For example, some elementary school children hold what Vosniadou and Brewer labeled a "dual-earth" theory. 
They believe that there are two earths: a flat one on which we live, and a spherical one in the sky.

Children do not simply take information they gain from a symbol and replace their preconceived notions about a concept. Instead, they actively integrate new and old information, and teachers must be sensitive to how seemingly inconsistent or even bizarre explanations (e.g., the dual earth theory) can arise as a consequence of what appear to children to be inconsistencies between what they learn from direct observation and what they learn from symbols.

\section{Conclusions}

The purpose of this brief review of the use of informational symbols in education has not been to argue that teachers should do away with informational symbols. Informational symbols can play an extremely important role in education, but they are not a panacea. Using a symbol to represent an abstract concept does not guarantee, or even necessarily increase the odds, that children will learn the concept.

The preceding examples illustrate that confusion about the nature and use of symbols is not limited to the very young children we have studied in our lab. Even school children can be confused and led astray by well-intentioned educational aids, if they do not fully understand how those aids relate to their referents.

As our examples illustrate, children can fail to comprehend several different aspects of symbol-referent relations. They can fail to realize that the symbol is a representation of something else in the first place. Young children watching the Sesame Street beautifulletter contest may not realize that what they are watching has anything to do with letters, even if they already know something about letters. More likely, they simply interpret the clothed and bejeweled shapes on the screen as standard cartoon characters with no other significance.

Symbols may also fail to be of assistance in an educational setting if children do not understand how the symbol is related to its referent. The children who were asked to use blocks to help them solve math problems may have understood that the blocks were relevant to the task at hand, but they were apparently unaware of how they were supposed to be used. In the case of beginning readers, they often assume that the symbol — printed words - will share some degree of physical similarity with its referent. Hence, they mistakenly expect the size and shape of the printed word to offer a clue as to its meaning.

Finally, even when children know that some entity is a representation for something else and understand in general how it is related to its referent, they can still have great difficulty understanding how to map from one to the other. The fascinating errors made by novice map readers illustrate that knowing something is a map is only the first step in being able to use the systematic relations among the elements on the map to interpret it.

We have particularly stressed that one variable that can complicate symbol use is the nature of the symbolic object itself. An object that has a salient identity and interest value of its own is in some ways more difficult to use as a symbol. This is especially true with respect to achieving the initial insight that something is a symbol, as in our model studies. It is apparently also true with respect to using a symbolic object. Hence, the approach of using a variety of different objects as manipulatives for teaching math may, as Stevenson \& Stigler (1992) have noted, be counterproductive. 
Care and thought are thus required to select symbolic objects that will in fact have the desired beneficial effect on learning. We should heed Langer (1942), who long ago noted that a peach is not a good symbol, because we care too much about peaches.

Acknowledgements-The research reported here was supported by a grant from the National Institute of Child Health and Human Development (HD-25271) to Judy DeLoache. Uttal and Pierroutsakos were also supported by a training grant from NICHD awarded to the Psychology Department of the University of Illinois. We thank Kathy Anderson, Nicole Bryant, Donald Marzolf, and Catherine Smith for their help with data collection. Thanks also to the children and staff of the Child Development Lab of the University of Illinois.

\section{References}

Bialystok, E. (1991). Letters, sounds, and symbols: Changes in children's understanding of written language. Applied Psycholinguistics, 12, 75-89.

Bialystok, E. (1992). Symbolic representation of letters and numbers. Cognitive Development, 7, 301-316.

Daehler, M. W., Lonardo, R., \& Bukatko, D. (1979). Matching and equivalence judgment in very young children. Child Development, 50, 170-179.

DeLoache, J. S. (1986). Memory in very young children: exploitation of cues to the location of a hidden object. Cognitive Development, 1, 123-137.

DeLoache, J. S. (1987). Rapid change in the symbolic functioning of very young children. Science, 238, $1556-1557$.

DeLoache, J. S. (1991). Symbolic functioning in very young children: understanding of pictures and models. Child Development, 62, 736-752.

DeLoache, J. S. (1995a). Early symbol understanding and use. In D. Medin (Ed.), The psychology of learning and motivation (Vol. 33, pp. 65-114).

DeLoache, J. S. (1995b). Early understanding and use of symbols: The model model. Current Directions in Psychological Science, 4, 109-113.

DeLoache, J. S., \& Burns, N. M. (1993). Symbolic development in young children: Understanding models and pictures. In C. Pratt \& A. F. Garton (Eds.), Systems of representation in children: Development and use (pp. 91-112). Chichester, England: Wiley.

DeLoache, J. S., \& Burns, N. M. (1994). Early understanding of the representational function of pictures. Cognition, 52, 83-110.

DeLoache, J. S., Kolstad, V., \& Anderson, K. N. (1991). Perceptual similarity and young children's understanding of scale models. Child Development, 62, 111-126.

DeLoache, J. S., Miller, K., \& Rosengren, K. (1997). The credible shrinking room: Very young children"s performance with symbolic and nonsymbolic relations. Psychological Science, 8, 308-313.

DeLoache, J.S., Pierroutsakos, S.L., Uttal, D.H., Rosengren, K., \& Gottlieb, A. (1998). Grasping the nature of pictures. Psychological Science, 9, 205-210.

DeLoache, J. S., Strauss, M. S., \& Maynard, J. (1979). Picture perception in infancy. Infant Behavior and Development, 2, 77-89.

Dirks, J., \& Gibson, E. (1977). Infants' perceptions of similarity between live people and their photographs. Child Development, 48, 124-130.

Goswami, U. (1992). Analogical reasoning in children. East Sussex, England: Lawrence Erlbaum.

Hughes, M. (1986). Children and number: Difficulties in learning mathematics. Oxford, England: Basil Blackwell.

Langer, S. K. K. (1942). Philosophy in a new key. Cambridge, MA: Harvard University Press.

Liben, L. S., \& Downs, R. M. (1989). Understanding maps as symbols: The development of map concepts in children. In H. W. Reese (Ed.), Advances in child development and behavior (Vol. 22, pp. 146-202). San Diego: Academic Press.

Liben, L. S., \& Downs, R. M. (1992). Developing an understanding of graphic representations in children and adults: The case of GEO-graphics. Cognitive Development, 7, 331-349. 
Murphy, C. M. (1978). Pointing in the context of shared activity. Child Development, 49, 371-380.

Ninio, A., \& Bruner, J. (1978). The achievement and antecedents of labelling. Journal of Child Language, 5, $1-15$.

Sigel, I. E. (1953). Developmental trends in the abstraction ability of children. Child Development, 24, 131-144.

Sigel, I. E., Anderson, L. M., \& Shapiro, H. (1966). Categorization behavior of lower and middle class Negro preschool children: differences in dealing with representations of familiar objects. Journal of Negro Education, $35,218-229$.

Stevenson, H. W., \& Stigler, J. W. (1992). The learning gap: Why our schools are failing and what we can learn from Japanese and Chinese education. New York: Summit Books.

Vosniadou, S., \& Brewer, W. F. (1992). Mental models of the earth: A study of conceptual change in childhood. Cognitive Psychology, 24, 535-585. 